\title{
Forecasting the Future Temperature Using a Downscaling Method by LARS-WG Stochastic Weather Generator at the Local Site of Phitsanulok Province, Thailand
}

\author{
Surasit Punyawansiri*, Bancha Kwanyuen \\ Department of Irrigation Engineering, Faculty of Engineering, Kasetsart University, Nakhon Pathom, Thailand \\ Email: ${ }^{\star}$ Surasit.Pu@ku.th
}

How to cite this paper: Punyawansiri, S. and Kwanyuen, B. (2020) Forecasting the Future Temperature Using a Downscaling Method by LARS-WG Stochastic Weather Generator at the Local Site of Phitsanulok Province, Thailand. Atmospheric and Climate Sciences, 10, 538-552.

https://doi.org/10.4236/acs.2020.104028

Received: August 12, 2020

Accepted: October 9, 2020

Published: October 12, 2020

Copyright $\odot 2020$ by author(s) and Scientific Research Publishing Inc. This work is licensed under the Creative Commons Attribution International License (CC BY 4.0).

http://creativecommons.org/licenses/by/4.0/

\section{Open Access}

\begin{abstract}
The study evaluates the effect of climate change on temperature, which is one of the most important variables in water resources management and irrigation scheduling. Climate prediction is necessary in the agricultural and hydrological analysis. This study proposed an approach to the application of the Long Ashton Research Station Weather Generator (LARS-WG) in Coupled Model Inter-comparison Project Phase 5 (CMIP5) under EC-Earth and MPI-ESM-MR. The first step is model calibration, where the observed dataset is analyzed statistically. In the second stage, the synthetic data and observed data are checked for Kolmogorov-Smirnov and the means and standard deviations. In order to evaluate the response of temperature under future warmer climate trends, the approach was assessed using data series. These parameters consisted of the minimum and maximum temperature at the Phitsanulok Meteorological Station (WMO Index 48378) and RCP4.5 climate change scenario for the base period as well as for 2021-2040 (the near future), 2041-2060 (the medium future) and 2061-2080 (the far future). The results of the numerical applications indicated that the linkage between the observed data spatially downscaled from LARS-WG simulations with the historical one of the locations during the baseline period had a very good accuracy. It was also found that the future climate change of temperature contributed to higher change. The mean of minimum temperature in the baseline year was $23.13^{\circ} \mathrm{C}$ while the mean of minimum temperature in the projection period for 2021-2040, 2041-2060 and 2061-2080 is expected to be 24.09 (+4.18\%), 24.49 $(+5.94 \%)$ and $24.82(+7.36 \%)^{\circ} \mathrm{C}$, and $24.12(+4.32 \%), 24.82(+7.36 \%)$ and $25.08(+8.48 \%)^{\circ} \mathrm{C}$ for the EC-Earth and MPI-ESM-MR, respectively. While, the mean of maximum temperature in the baseline year was $33.41^{\circ} \mathrm{C}$, the
\end{abstract}


maximum temperatures are projected to increase at $34.47(+3.19 \%), 34.88$ $(+4.43 \%)$ and $35.21(+5.40 \%)^{\circ} \mathrm{C}$, and $34.53(+3.36 \%), 35.19(+5.34 \%)$ and $35.30(+5.67 \%)^{\circ} \mathrm{C}$, respectively. Furthermore, the future local surface temperatures from the MPI-ESM-MR project tended to be higher than EC-Earth. In conclusion, the study results indicate that in coming three time periods, the minimum and maximum temperature increase is expected in Phitsanulok province, Thailand.

\section{Keywords}

LARS-WG, CMIP5, Climate Change, Downscaling, Temperature

\section{Introduction}

According to existing evidence as well as the fluctuating influences of greenhouse gases and human activities, climate is a complicated system that is continuously evolving. Appropriate approaches for decreasing the fatal effects of climate change phenomena are possible by forecasting and examining the rate of change in meteorological considerations. Employing modelled climatic variables by General Circulation Models or Global Climate Models (GCMs) is one of the most operative methods for assessing the influences of climate change occurrences [1] [2]. These simulations can forecast the changes resulting from the impact of greenhouse gasses on weather conditions. The output of these models should be reduced when they show low spatial resolution power. Awareness of potential climate change leads to the adoption of mitigation strategies, as these effects have implications for areas of public interest including agriculture [3] [4], hydrological [5] [6] and water management [7] [8].

The IPCC describes GCMs as a computational (quantitative) description of the climate system on the basis of the physical, chemical and biological characteristics of its elements, their correlations and feedback mechanisms [9]. GCMs, centered on statistical models of the atmosphere, ocean and land surface systems, are thought to be the only reliable instruments presently offered to model the reaction of the global climate system to rising greenhouse gas levels. Because of the restricted depiction of scale atmospheric processes, topography and land-sea distribution in GCMs, however, the direct use of output from GCMs is frequently insufficient [10].

Potential climate forecasts of GCMs in various conditions are typically used to establish climate mitigation approaches. Current GCMs still have insufficient ability to predict complex and local climate characteristics or provide accurate information on temperature and rainfall, which is the key input for hydrological effect evaluation criteria. Additionally, the contributions given by GCMs are too imprecise to be used by these hydrological models as they involve details at finer scales. Downscaled GCM results are typically used to present the fine-resolution or point-scale information needed for impact simulations [11]. 
A computer algorithm that incorporates weather and climate records to generate a long series of synthetic daily weather data of continuous integration for a location due to the statistical features of the studied weather data for that location is a stochastic weather generator. One of the approaches for advancing local scale future climate situations from large-scale climate changes modelled by GCMs is stochastic weather generators [12]. Recently, establishing the prospective influence on hydrological applications has been carried out in climate change and variability studies using simulated weather data created by weather generators [13] [14].

These selected weather generators have been used in different climate change impact studies, including studies with LARS-WG weather generators [15]. The current version 6.0 of LARS-WG incorporates climate projections from the CMIP5 ensemble used in the IPCC Fifth Assessment Report (AR5) [16]. Previous version of LARS-WG 5.5 which climate projections from the CMIP3 provided the scientific base for the Fourth Assessment Report (AR4) of IPCC published in 2007 [17]. The LARS-WG 6.0 has been well validated in diverse climates around the world. The LARS-WG version 6.0 can be download from https://www.rothamsted.ac.uk/, the link for downloading LARS-WG 6.0 is given in https://sites.google.com/view/lars-wg/.

The objectives of this study were to use the new version of the LARS-WG in predicting climate change for the periods 2021-2040 (the near future), 2041-2060, (the medium future), and 2061-2080 (the far future) according to the projections of EC-Earth and MPI-ESM-MR GCMs for RCP4.5 scenarios (medium greenhouse gas emission scenario) at the local site of Phitsanulok province, Northern of Thailand.

\section{Data and Methods}

\subsection{The Study Area}

The study site was Phitsanulok Meteorological Station (PMS) which is located at latitude $16.783^{\circ} \mathrm{N}$, longitude $100.267^{\circ} \mathrm{E}$ and altitude 44 meters above mean sea level, as shown in Figure 1. This area supplies much of the agricultural produce of Thailand, and is under intense demand pressures regarding climate change for agricultural and irrigation system.

The site is influenced by the tropical region which has two seasons: the dry season from November to April, and the rainy season from May to October. The average minimum and maximum temperature were $22.73^{\circ} \mathrm{C}, 33.26^{\circ} \mathrm{C}$ in $1961-$ 2019.

Historical climate data for daily precipitation, the minimum and maximum temperature were obtained from PMS (WMO Index 48378) for the period 1961-2019. Only data for the period 1980-2010 (the baseline year) were selected for analysis as required for the calibration and validation of the weather generator. The weather data did not include solar radiation. The climate data of $3.17 \%$ were missing from the observed station data. Missing data in the observed data- 
set may alter the statistics, but the long-term climate series will still be generated, missing data values should be coded -99.0 of the layout of a typical file of weather data for use in LARS-WG [18]. The generator outputs result of statistical tests comparing the observed and generated data.

\subsection{LARS-WG Stochastic Weather Generator}

LARS-WG is a stochastic weather generator which could be utilized to predict weather data at a single station under both present and potential atmospheric conditions. The data comprises the regular time series for a number of environmental factors, including rainfall, maximum temperature and minimum temperature, and solar radiation. LARS-WG employs a semi-empirical distribution (SED) known as the cumulative probability distribution function $(\mathrm{CDF})$ to estimate the confidence interval for minimum and maximum temperatures.

$$
E P M=\left\{a_{0}, a_{i}, h_{i}, i=0 \ldots, 23\right\} .
$$

EPM is a distribution histogram of 23 distinct intervals $\left(a_{i-1}, a_{i}\right)$ where $a_{i-1}<a_{i}$ and gives a more precise picture of the observable distribution compared to 10 used in the earlier models. By disrupting the distribution parameters for a site with projected climate change from global or regional climate simulations, a regular environment model for this site may be created and used in tandem with a process-based impact assessment model. Generally, the approach for producing synthetic weather data can be divided into three different steps: model initialization, model validation, and scenario creation, as described and briefly defined below [19].

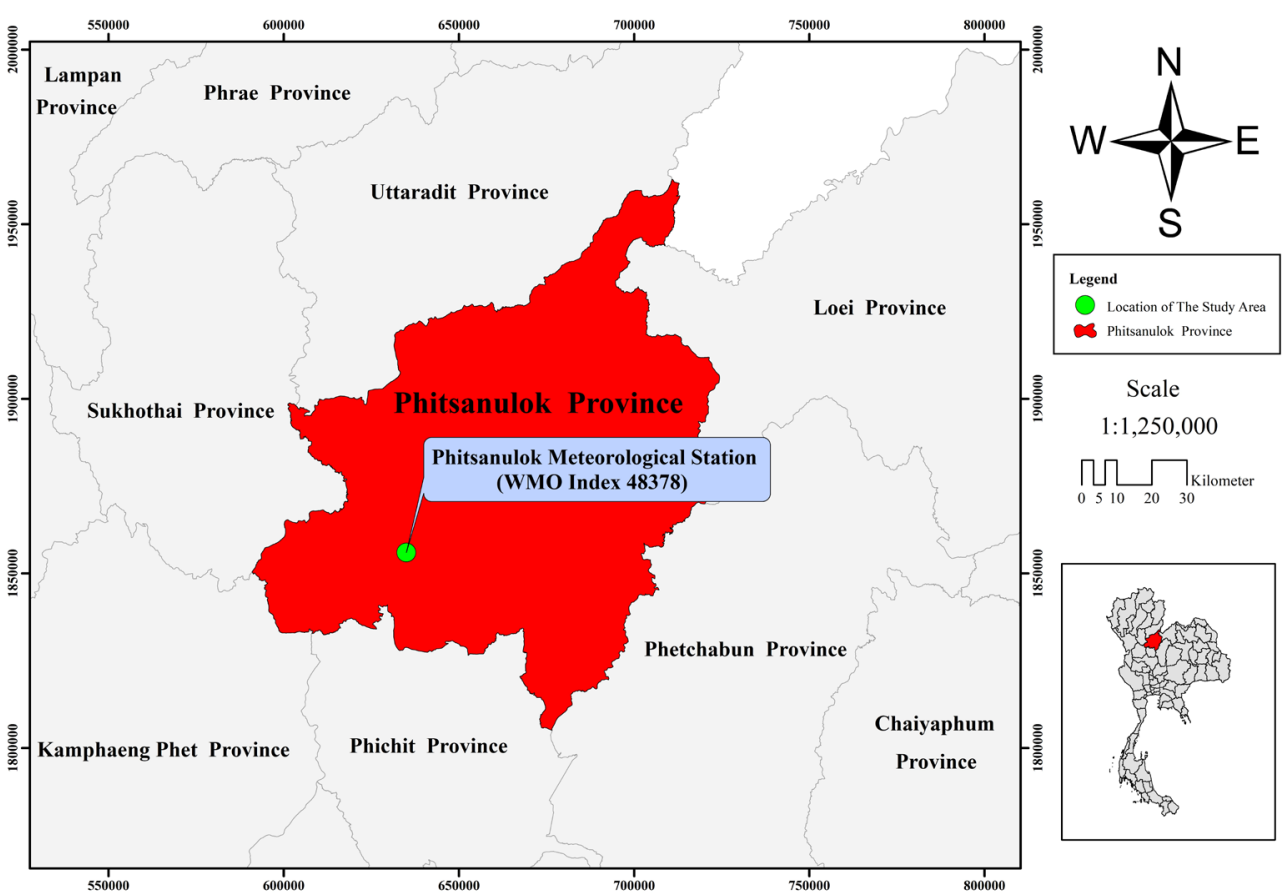

Figure 1. Location of the study area. 
In the case of the minimum and maximum temperature two values close to 0 and 1 are used to interpret for extremely low and high temperatures, i.e. $p_{2}=$ $0.01, p_{3}=0.02, p_{n-1}=0.99$ and $p_{n-2}=0.98$. Both values of $p i(0<I<n)$. For the latest edition of LARS-WG 6.0, the low and high temperatures for dry and rainy days are estimated by SEDs measured for each month [20].

The contribution to LARS-WG is a collection of seasonal data (rainfall, low temperature and high temperature) from the base time (1980-2010) and site data (latitude, longitude and altitude). The perceived daily weather data at a given site was employed to establish a set of parameters for the probability distributions of weather variables after input data preparation and quality control. Such criteria are used to produce a simulated weather sequence of variable lengths by randomly choosing values from the suitable distributions, with the same statistical properties as the original data observed, but varying on a day-to-day level. LARS-WG determines wet days from dry days depending on whether or not rainfall is zero. Alternating wet and dry series estimated by semi-empirical probability distributions are used to model the existence of rainfall. The statistical properties of the actual and simulated weather data during the model testing were evaluated to assess whether there were any statistically important variations using the Chi-square strength test (Kolmogorov-Smirnov, KS) and the means and standard deviations using the $\mathrm{t}$ and $\mathrm{F}$ measures, respectively. By varying the constraints of LARS-WG, number of years and seed numbers can be conducted. The LARS-WG baseline constraints, which are analyzed from the perceived weather for a baseline period (1980-2010) are regulated by the delta-changes for the future period and the emissions projected by a GCM for each climatic variable covering the site for creation of climate scenarios at the site for a particular time in the future with a designated emissions scenario.

In this study, the local-scale climate scenarios based on RCP4.5 scenarios simulated by the selected two GCMs are generated for the time periods of 2021-2040, 2041-2060 and 2061-2080 to predict the future change of temperature in PMS. The coefficient of determination for a linear regression model is the quotient of the variances of the generated data and observed data values [21]. The coefficient of determination is computed according to the Equation (2) below:

$$
R^{2}=\frac{\sum(y-\bar{y})^{2}-\sum(y-\hat{y})^{2}}{\sum(y-\bar{y})^{2}}
$$

The coefficient of determination $\left(R^{2}\right)$, represents the proportion of the total sample variation in $y$ (measured by the sum of squares of deviations of the sample $y$ values about their mean $y$ ) which is explained by the linear relationship between generated data and observed data.

\subsection{Global Climate Models (GCMs)}

The new version of the LARS-WG 6.0 was applied for this study that incorporated predictions from two GCMs, which were used in the IPCC's Fifth Assessment Report (AR5) based on Emissions Scenarios RCP4.5 (medium greenhouse 
gas emission scenario) for three times the period as listed in Table 1. These two models differ in spatial resolution power, design institute, predictability of atmospheric variables, and predictability of oceanic variables [22] [23]. The simulation of GCMs assumes the year length is 365 calendar days, with global coverage and daily.

\section{Results and Discussion}

\subsection{Calibration and Validation of LARS-WG}

The "Site Analysis" function in the LARS-WG model with two data sets, comprising observed station and GCMs data, were utilized to conduct the calibration and validation. The Kolmogorov-Smirnov test, F-test and t-test were operated to assess the functioning of the weather generator during calibration and validation. To assess the similarity of distributions for daily maximum and minimum temperature, the KS test was used. The equivalence of 30-day mean of daily maximum temperature (Tmax) and the 30-day mean of daily minimum temperature (Tmin) were assessed by the F-test. Both experiments measure a p-value that is used to support or dismiss the hypothesis that the two data sets (detected and created) may have been obtained from the same distribution at a 5 percent level of significance.

The coefficient of determinant $\left(R^{2}\right)$ was used to check the performance. Tables 2-6 show the estimation of suitability for LARS-WG function in replicating for PMS. The model executed very well in fitting for the two datasets, as shown by the KS test. LARS-WG performance was effective in simulating both minimum and maximum temperature for both data sets, as revealed by the assessment results.

Figures 2-5 show the relationship linking the 30-day mean and standard deviation of temperature for the two data sets employed in the study. For measuring the 30-day means of Tmin and Tmax statistics, the outcomes displayed excellent performance by LARS-WG. Using LARS-WG, the mean periodic totals for the minimum and maximum temperatures were formed efficiently, which showed that the temperature can be analyzed using the daily time series. The findings of the experimental analyses showed that there were no major variations in the calculated mean of the measured average temperature relative to the estimates. LARS-WG precisely created the 30-day means of minimum and maximum temperature values, offering a coefficient of determinants equivalent to units. As shown in Table 2, the $R^{2}$ for the mean 30-day minimum and maximum temperature had a robust linear correlation linking the experimental, created and artificial data.

Table 1. The global climate models from IPCC AR5.

\begin{tabular}{ccc}
\hline The Global Climate Models & Country and Institution developed the GCMs & Resolution \\
\hline EC-Earth & EC-Earth consortium, Europe & $1.00^{\circ} \times 1.00^{\circ}$ \\
MPI-ESM-MR & Max-Planck Institute for Meteorology, Germany & $1.875^{\circ} \times 1.875^{\circ}$ \\
\hline
\end{tabular}


Table 2. Coefficient of determination $\left(R^{2}\right)$ in calibration.

\begin{tabular}{ccc}
\hline Temperature & Observed data & Generated data \\
\hline Tmin & $99.7 \%$ & $99.5 \%$ \\
Tmax & $99.6 \%$ & $99.5 \%$ \\
\hline
\end{tabular}

Table 3. KS-test of daily distributions for observed data.

\begin{tabular}{ccccccc}
\hline \multirow{2}{*}{ Month } & \multicolumn{2}{c}{ Tmin } & & & Tmax & Assess \\
\cline { 2 - 6 } & KS & p-value & Assess & KS & p-value & Perfect fit \\
January & 0.106 & 0.999 & Perfect fit & 0.106 & 0.999 & Perfect fit \\
February & 0.106 & 0.999 & Perfect fit & 0.106 & 0.999 & Perfect fit \\
March & 0.053 & 1.000 & Perfect fit & 0.106 & 0.999 & Perfect fit \\
April & 0.106 & 0.999 & Perfect fit & 0.106 & 0.999 & Perfect fit \\
May & 0.053 & 1.000 & Perfect fit & 0.158 & 0.913 & Perfect fit \\
June & 0.053 & 1.000 & Perfect fit & 0.053 & 1.000 & Perfect fit \\
July & 0.053 & 1.000 & Perfect fit & 0.053 & 1.000 & Perfect fit \\
August & 0.053 & 1.000 & Perfect fit & 0.053 & 1.000 & Perfect fit \\
September & 0.053 & 1.000 & Perfect fit & 0.106 & 0.999 & Perfect fit \\
October & 0.106 & 0.999 & Perfect fit & 0.053 & 1.000 & Perfect fit \\
November & 0.053 & 1.000 & Perfect fit & 0.105 & 0.999 & 1.000 \\
December & 0.053 & 1.000 & Perfect fit & 0.053 & & Perfect fit \\
\hline
\end{tabular}

Table 4. KS-test of daily distributions for generated data.

\begin{tabular}{|c|c|c|c|c|c|c|}
\hline \multirow{2}{*}{ Month } & \multicolumn{3}{|c|}{ Tmin } & \multicolumn{3}{|c|}{$\operatorname{Tmax}$} \\
\hline & KS & p-value & Assess & KS & $\mathrm{p}$-value & Assess \\
\hline January & 0.053 & 1.000 & Perfect fit & 0.053 & 1.000 & Perfect fit \\
\hline February & 0.053 & 1.000 & Perfect fit & 0.106 & 0.999 & Perfect fit \\
\hline March & 0.053 & 1.000 & Perfect fit & 0.053 & 1.000 & Perfect fit \\
\hline April & 0.106 & 0.999 & Perfect fit & 0.053 & 1.000 & Perfect fit \\
\hline May & 0.053 & 1.000 & Perfect fit & 0.053 & 1.000 & Perfect fit \\
\hline June & 0.053 & 1.000 & Perfect fit & 0.053 & 1.000 & Perfect fit \\
\hline July & 0.053 & 1.000 & Perfect fit & 0.033 & 1.000 & Perfect fit \\
\hline August & 0.053 & 1.000 & Perfect fit & 0.053 & 1.000 & Perfect fit \\
\hline September & 0.053 & 1.000 & Perfect fit & 0.053 & 1.000 & Perfect fit \\
\hline October & 0.053 & 1.000 & Perfect fit & 0.053 & 1.000 & Perfect fit \\
\hline November & 0.053 & 1.000 & Perfect fit & 0.053 & 1.000 & Perfect fit \\
\hline December & 0.053 & 1.000 & Perfect fit & 0.053 & 1.000 & Perfect fit \\
\hline
\end{tabular}


Table 5. Future mean of minimum temperature $\left({ }^{\circ} \mathrm{C}\right)$ under RCP4.5 scenarios.

\begin{tabular}{|c|c|c|c|c|c|c|c|}
\hline \multirow{2}{*}{ Month } & \multirow{2}{*}{ Base $1980-2010$} & \multicolumn{3}{|c|}{ EC-Earth } & \multicolumn{3}{|c|}{ MPI-ESM } \\
\hline & & 2030 s & $2050 s$ & $2070 \mathrm{~s}$ & $2030 s$ & $2050 \mathrm{~s}$ & $2070 \mathrm{~s}$ \\
\hline January & 18.68 & 19.77 & 20.53 & 20.74 & 19.47 & 20.70 & 20.90 \\
\hline February & 20.94 & 21.73 & 22.23 & 22.64 & 21.37 & 22.57 & 22.60 \\
\hline March & 23.60 & 25.07 & 25.17 & 25.56 & 24.68 & 25.78 & 25.69 \\
\hline April & 25.35 & 26.74 & 26.52 & 26.94 & 26.41 & 27.43 & 27.34 \\
\hline May & 25.17 & 26.25 & 26.55 & 26.99 & 26.49 & 27.16 & 27.36 \\
\hline June & 25.02 & 26.03 & 26.79 & 26.99 & 26.46 & 26.96 & 27.24 \\
\hline July & 24.77 & 25.69 & 26.36 & 26.58 & 26.07 & 26.40 & 26.71 \\
\hline August & 24.69 & 25.49 & 25.85 & 26.29 & 25.82 & 26.11 & 26.37 \\
\hline September & 24.68 & 25.41 & 25.55 & 26.07 & 25.55 & 25.96 & 26.18 \\
\hline October & 24.10 & 24.79 & 25.05 & 25.40 & 24.75 & 25.26 & 25.65 \\
\hline November & 21.76 & 22.55 & 23.05 & 23.27 & 22.61 & 23.14 & 23.79 \\
\hline December & 18.66 & 19.50 & 20.26 & 20.36 & 19.73 & 20.37 & 21.12 \\
\hline
\end{tabular}

Table 6. Future mean of maximum temperature $\left({ }^{\circ} \mathrm{C}\right)$ under RCP4.5 scenarios.

\begin{tabular}{|c|c|c|c|c|c|c|c|}
\hline \multirow{2}{*}{ Month } & \multirow{2}{*}{ Base $1980-2010$} & \multicolumn{3}{|c|}{ EC-Earth } & \multicolumn{3}{|c|}{ MPI-ESM } \\
\hline & & 2030 s & $2050 s$ & $2070 \mathrm{~s}$ & $2030 s$ & 2050 s & $2070 \mathrm{~s}$ \\
\hline January & 31.70 & 33.05 & 33.81 & 34.01 & 32.86 & 34.15 & 34.20 \\
\hline February & 33.75 & 34.86 & 35.36 & 35.77 & 34.43 & 35.82 & 35.46 \\
\hline March & 35.73 & 37.29 & 37.39 & 37.78 & 36.70 & 37.92 & 37.41 \\
\hline April & 37.19 & 38.54 & 38.42 & 38.84 & 38.08 & 38.92 & 38.67 \\
\hline May & 35.51 & 36.58 & 36.88 & 37.31 & 36.83 & 37.30 & 37.41 \\
\hline June & 33.94 & 35.03 & 35.75 & 35.97 & 35.75 & 35.96 & 36.23 \\
\hline July & 33.18 & 34.21 & 34.88 & 35.10 & 34.96 & 34.95 & 35.18 \\
\hline August & 32.53 & 33.52 & 33.87 & 34.32 & 34.05 & 34.04 & 34.22 \\
\hline September & 32.48 & 33.24 & 33.37 & 33.91 & 33.37 & 33.62 & 33.82 \\
\hline October & 32.38 & 33.22 & 33.52 & 33.83 & 33.09 & 33.65 & 34.08 \\
\hline November & 31.74 & 32.56 & 33.06 & 33.28 & 32.57 & 33.31 & 33.85 \\
\hline December & 30.73 & 31.53 & 32.30 & 32.39 & 31.64 & 32.62 & 33.05 \\
\hline
\end{tabular}

Compared to those calculated from the experimental time series, the means and variances of day-to-day artificial weather data were assumed to be insignificantly distinctive. It is likewise critical that the artificial weather series adopt a confidence interval that is not significantly varied from the actual time series. As seen in Figure 4, Figure 5, the 30-day temperature standard deviations for the created values were miscalculated for both data sets (perceived and produced). The evaluation results exhibit that LARS-WG execution in reproducing the distributions of the day-to-day temperature in all months is ideal, as seen from the results in Table 3 and Table 4. 

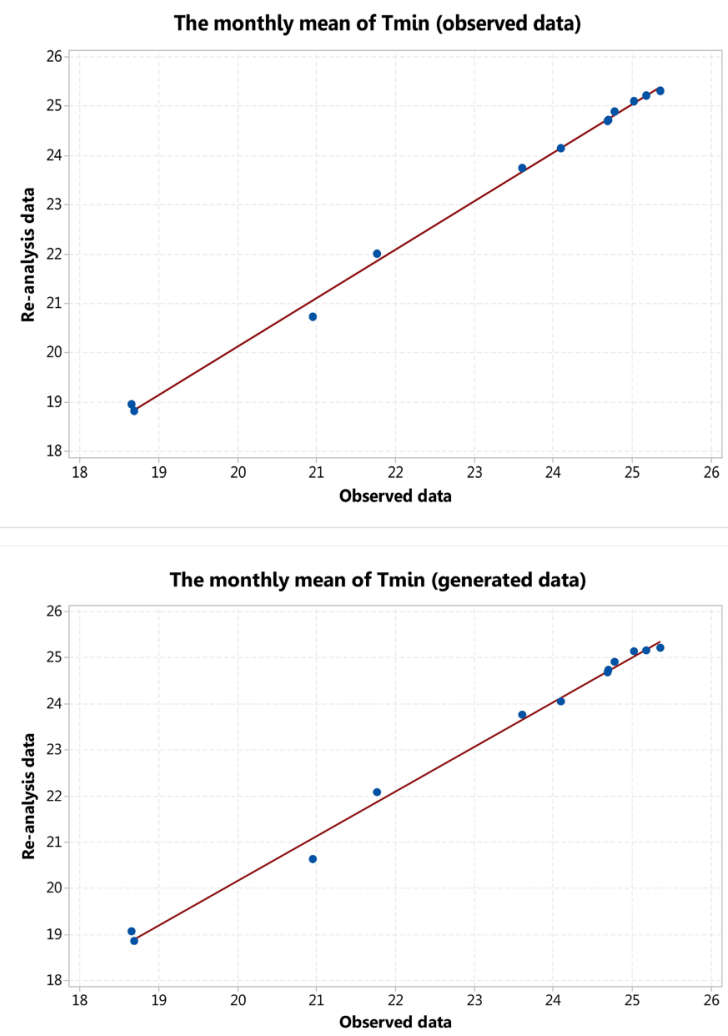

Figure 2. Tmin monthly mean of observed versus generated data.
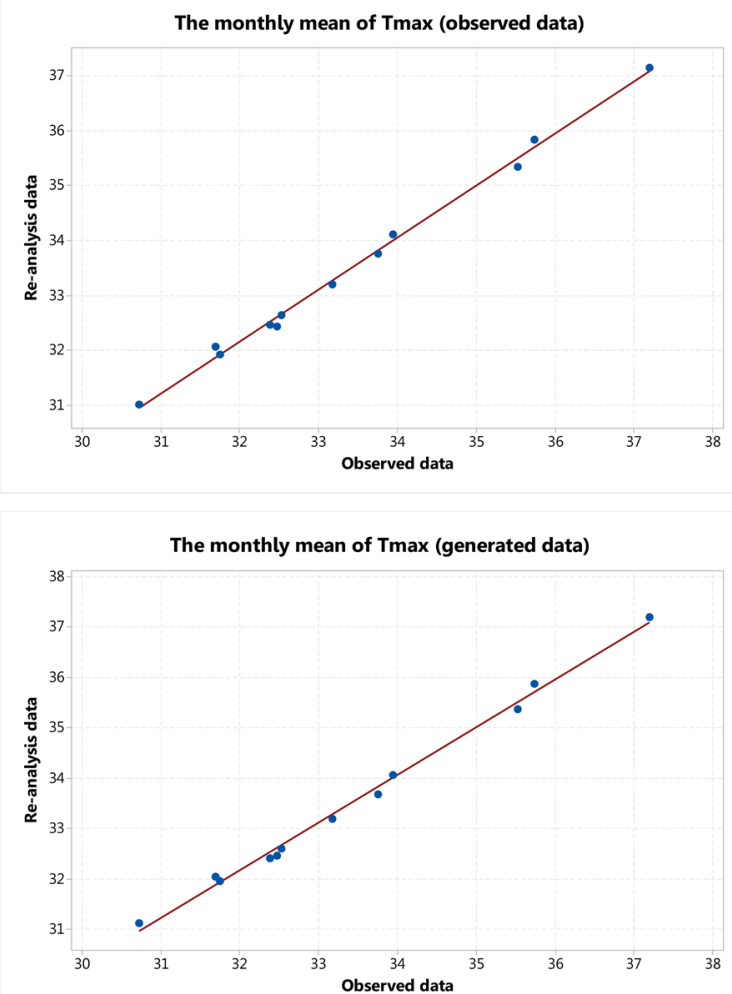

Figure 3. Tmax monthly mean of observed versus generated data. 

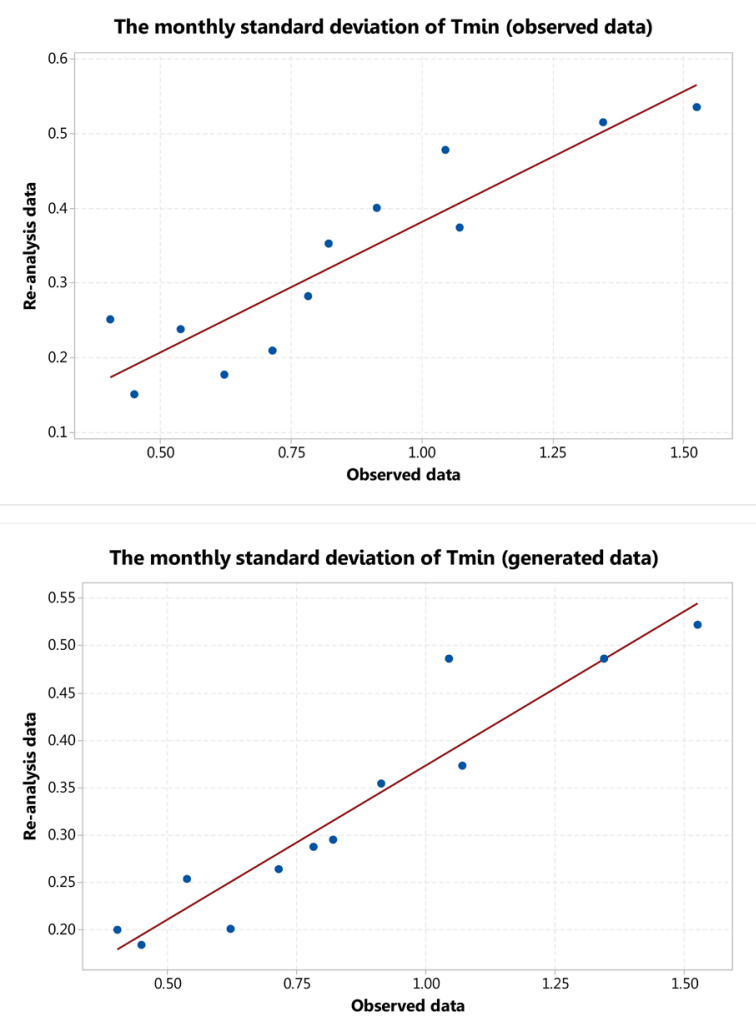

Figure 4. Tmin monthly standard deviations of observed versus generated data.
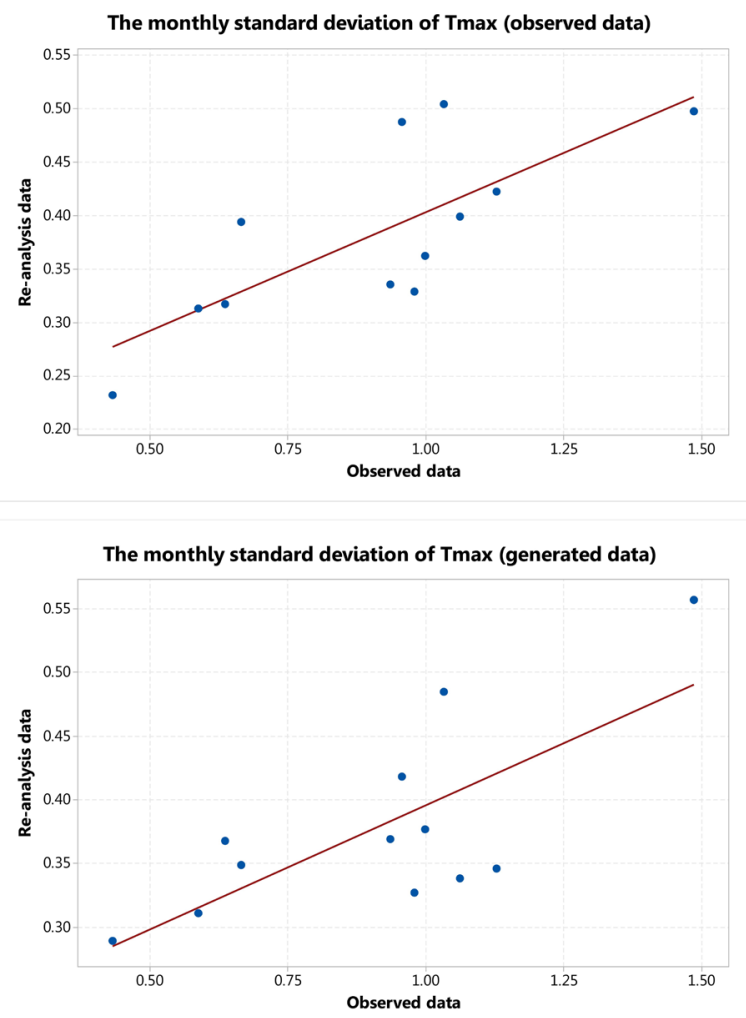

Figure 5. Tmax monthly standard deviations of observed versus generated data. 


\subsection{Future Scenarios of Minimum and Maximum Temperature}

The EC-Earth and MPI-ESM-MR GCMs and RCP4.5 scenarios in LARS-WG version 6.0 were used in this study to generate future climate scenarios to better deal with un-certainties. In Figure 6, Figure 7, graphs of the temporal evolution of the indicator Tmin and Tmax in the PMS part of Phitsanulok Province of Thailand are presented. Table 5, Table 6 show the mean of Tmin and Tmax in base time (1990s) would be 23.12 and $33.41\left({ }^{\circ} \mathrm{C}\right)$. The Tmin would increase $\left({ }^{\circ} \mathrm{C}\right)$ by $0.97,1.37$ and 1.70 under EC-Earth RCP4.5 (2030s), RCP4.5 (2050s) and RCP4.5 (2070s), respectively. From the MPI-ESM-MR, results showed the increase $\left({ }^{\circ} \mathrm{C}\right)$ in the mean Tmin by 1.00 under RCP4.5 (2030s) after that an increase in the mean of Tmin by 1.70 and 1.96 under RCP4.5 (2050s) and RCP4.5 (2070s), respectively.

The mean of Tmax would increase $\left({ }^{\circ} \mathrm{C}\right)$ by $1.06,1.48$, and 1.80 under ECEarth RCP4.5 (2030s), RCP4.5 (2050s) and RCP4.5 (2070s), respectively. While the MPI-ESM-MR, results showed the increase $\left({ }^{\circ} \mathrm{C}\right)$ in the mean of Tmax by 1.12, 1.78 and 1.89 under RCP4.5 (2030s), RCP4.5 (2050s) and RCP4.5 (2070s), as in Table 6. The changes in temperature are $0.97^{\circ} \mathrm{C}-1.80^{\circ} \mathrm{C}$ (EC-Earth), $1.00^{\circ} \mathrm{C}-1.89^{\circ} \mathrm{C}$ (MPI-ESM-MR) under RCP4.5 scenarios generated using the observed station data.

(A)

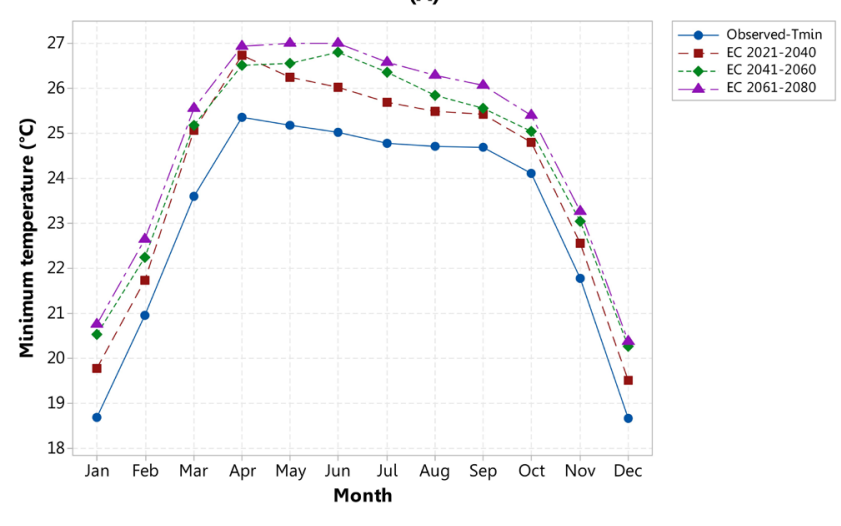

(B)

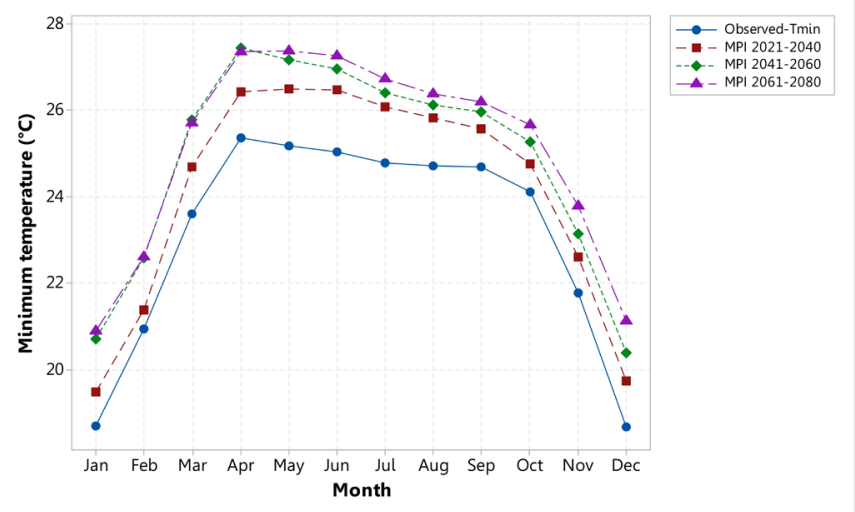

Figure 6. Baseline and future of minimum temperature (A) EC-Earth, (B) MPI-ESM-MR. 
(A)

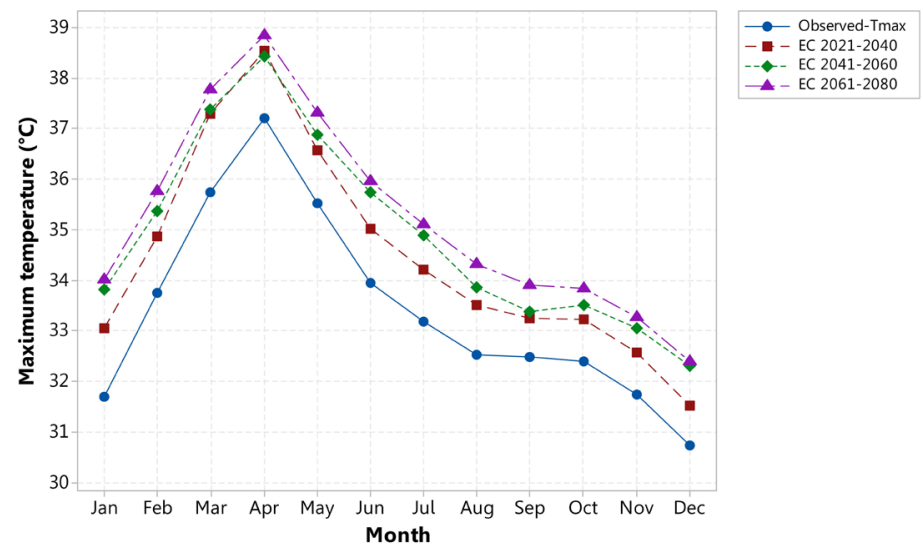

(B)

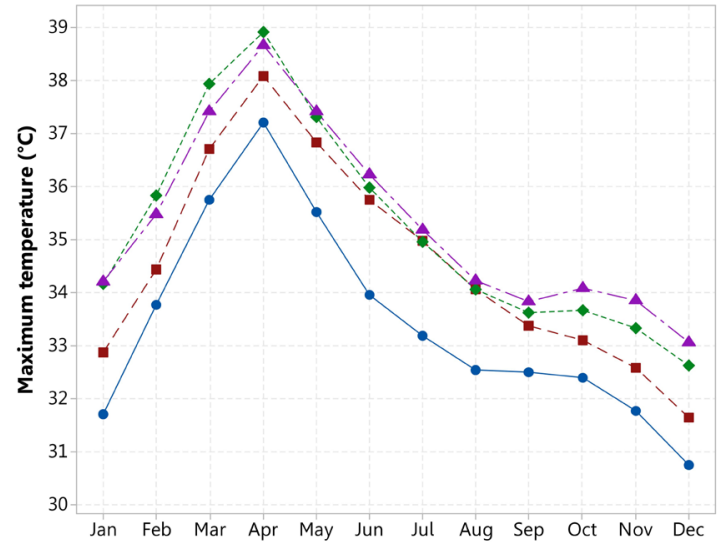

Figure 7. Baseline and future of maximum temperature (A) EC-Earth, (B) MPI-ESM-MR.

A step towards a widespread evaluation of uncertainty in future climate predictions is possible with the multi-ensembles method using various climate models and emissions circumstances. Nonetheless, the relative change factors (RCFs), resulting from the direct GCM output of the variables that precisely match the estimates, are used by the LARS-WG. Therefore, the reality that GCMs are not very accurate when replicating temperature means that the error caused by the GCM output for temperature extends the error of downscaling that causes the efficiency of LARS-WG to downscale temperature to demand more vigilance [24].

\section{Conclusion}

Using a LARS-WG 6.0 stochastic weather generator, this study replicated the meteoro-logical constraints from Phitsanulok meteorological station (minimum and maximum temperature). The findings revealed that these criteria were modelled with very high precision. LARS-WG may be used to produce climate mod- 
els for present and future PMS simulations. LARS-WG projected the 30-day mean low and high temperatures, which are consistent with the similarity between the observable and produced mean values of 0.999 and 0.998 respectively. The low and high temperature for PMS would rise in the 2030s, 2050s and 2070s under RCP4.5, as revealed by the results. Climate signals for temperature values in the 2030s, 2050s, and 2070s would rise under perceived station data, as suggested by the ensemble mean for the EC-Earth and MPI-ESM-MR GCMs. Further, impending local surface temperatures from the MPI-ESM-MR project were likely to be superior to EC-Earth. It was concluded from research that the LARS-WG model had the reasonable ability to imitate the day-to-day and 30-day temperature constraints and could be used as an operative tool for integrating climate change impacts into viable improvement. The work presented in this paper strongly supports the use of multi-GCMs model downscaling for providing the required data for agricultural and hydrological analysis. Additionally, we found that one of the many reasons for the large differences of the future minimum and maximum temperature is the CMIP5 models' resolution. Finally, the LARS-WG 6.0 provides a means of synthesizing long-term climate data that can then be used in impact of climate change assessment. Evaluate the performance of the generators for multi-GCMs and climate models are required to provide perspective into downscaling model vulnerabilities in order to establish alternate mitigation and adaptation approaches in climate change.

\section{Acknowledgements}

The authors would like to thank the Agricultural Research Development Agency, Thailand for supporting the study. The researchers also wish to thank Prof. Mikhail A. Semenov for the provision of Long Ashton Research Station Weather Generator, the license used in this study, and the Thai Meteorological Department (TMD) for providing the weather data.

\section{Conflicts of Interest}

The authors declare no conflicts of interest regarding the publication of this paper.

\section{References}

[1] Xuan, W., Chong Ma, C., Kang, L., Gu, H., Pan, S. and Xu, Y.P. (2017) Evaluating Historical Simulations of CMIP5 GCMs for Key Climatic Variables in Zhejiang Province, China. Theoretical and Applied Climatology, 128, 207-222. https://doi.org/10.1007/s00704-015-1704-7

[2] Kamworapan, S., Surussavadee, C. (2019) Evaluation of CMIP5 Global Climate Models for Simulating Climatological Temperature and Precipitation for Southeast Asia. Advances in Meteorology, 2019, Article ID: 1067365. https://doi.org/10.1155/2019/1067365

[3] Lesk, C., Rowhani, P. and Ramankutty, N. (2016) Influence of Extreme Weather Disasters on Global Crop Production. Nature, 529, 84-87. 
https://doi.org/10.1038/nature16467

[4] Masud, M.B., Ferdous, J. and Faramarzi, M. (2018) Projected Changes in Hydrological Variables in the Agricultural Region of Alberta, Canada. Water, 10, 1810. https://doi.org/10.3390/w10121810

[5] Moazzam, M.F.U., Lee, B.G., Rahman, G. and Waqas, T. (2020) Spatial Rainfall Variability and an Increasing Threat of Drought, According to Climate Change in Uttaradit Province, Thailand. Atmospheric and Climate Sciences, 10, 357-371. https://doi.org/10.4236/acs.2020.103020

[6] Manfreda, S., Iacobellis, V., Gioia, A., Fiorentino, M. and Kochanek, K. (2018) The Impact of Climate on Hydrological Extremes. Water, 10, 802.

https://doi.org/10.3390/w10060802

[7] Arnell, N.W. and Gosling, S.N. (2016) The Impacts of Climate Change on River Flood Risk at the Global Scale. Climatic Change, 134, 387-401.

https://doi.org/10.1007/s10584-014-1084-5

[8] Hattermann, F.F., Krysanova, V., Gosling, S.N., et al. (2017) Cross-Scale Intercomparison of Climate Change Impacts Simulated by Regional and Global Hydrological Models in Eleven Large River Basins. Climatic Change, 141, 561-576. https://doi.org/10.1007/s10584-016-1829-4

[9] IPCC Fourth Assessment Report (2007) Climate Change 2007: The Physical Science Basis :Contribution of Working Group I to the Fourth Assessment Report of the Intergovernmental Panel on Climate Change. Cambridge University Press, New York.

[10] Basharin, D., Polonsky, A. and Stankūnavičius, G. (2016) Projected Precipitation and Air Temperature over Europe Using a Performance-Based Selection Method of CMIP5 GCMs. Journal of Water and Climate Change, 7, 103-113.

https://doi.org/10.2166/wcc.2015.081

[11] Mcsweeney, C.F., Jones, R.G., Lee, R.W. and Rowell, D.P. (2015) Selecting CMIP5 GCMs for Downscaling over Multiple Regions. Climate Dynamics, 44, 3237-3260. https://doi.org/10.1007/s00382-014-2418-8

[12] Semenov, M.A. and Barrow, E.M. (1997) Use of a Stochastic Weather Generator in the Development of Climate Change Scenarios. Climatic Change, 35, 397-414. https://doi.org/10.1023/A:1005342632279

[13] Rajith Mukundan, R., Acharya, N., Gelda, R.K., Frei, A. and Owens, E.M. (2019) Modeling Streamflow Sensitivity to Climate Change in New Yorkcity Water Supply Streams Using a Stochastic Weather Generator. Journal of Hydrology. Regional Studies, 21, 147-158. https://doi.org/10.1016/j.ejrh.2019.01.001

[14] Kinnell, P.I.A. and Yu, B. (2020) CLIGEN as a Weather Generator for Predicting Rainfall Erosion Using USLE Based Modelling Systems. Catena, 194, Article ID: 104745. https://doi.org/10.1016/j.catena.2020.104745

[15] Bayatvarkeshi, M., Zhang, B., Fasihi, R., Adnan, R.M., Kisi, O. and Yuan, X. (2020) Investigation Into the Effects of Climate Change on Reference Evapotranspiration Using the Hadcm 3 and LARS-WG. Water, 12, 666.

[16] Field, C.B., Barros, V.R., Dokken D.J., et al. (2014) Climate Change 2014: Impacts, Adaptation, and Vulnerability. Part A: Global and Sectoral Aspects. Contribution of Working Group II to the Fifth Assessment Report of the Intergovernmental Panel on Climate Change, IPCC, Cambridge University Press, Cambridge.

[17] Disasa, K.N., Tura, F.S. and Fereda, M.E. (2019) Climate Change Downscaling Using Stochastic Weather Generator Model in Rift Valley Basins of Ethiopia. American Journal of Climate Change, 8, 561-590. https://doi.org/10.4236/ajcc.2019.84030 
[18] Semenov, M.A. and Barrow, E.M. (2002) LARS-WG a Stochastic Weather Generator for Use in Climate Impact Studies. User Manual, Hertfordshire.

[19] Racsko, P., Szeidl, L. and Semenov, M.A. (1991) A Serial Approach to Local Stochastic Weather Models. Ecological Modelling, 57, 27-41.

https://doi.org/10.1016/0304-3800(91)90053-4

[20] Semenov, M.A. (2007) Development of High-Resolution UKCIP02-Based Climate Change Scenarios in the UK. Agricultural and Forest Meteorology, 144, 127-138. https://doi.org/10.1016/j.agrformet.2007.02.003

[21] Fosco, M.V., Livia, P., Ermes, M., Gianni, B. and Roberto, C. (2019) Quantifying Uncertainty Due to Stochastic Weather Generators in Climate Change Impact Studies. Scientific Reports, 9, Article No. 9258.

https://doi.org/10.1038/s41598-019-45745-4

[22] Mcsweeney, C.F., Jones, R.G. and Lee, R.W. (2015) Selecting CMIP5 GCMs for Downscaling over Multiple Regions. Climate Dynamics, 44, 3237-3260. https://doi.org/10.1007/s00382-014-2418-8

[23] Xuan, W., Ma, C., Kang, L., Gu, H., Pan, S. and Xu, Y.P. (2017) Evaluating Historical Simulations of CMIP5 GCMs for Key Climatic Variables in Zhejiang Province, China. Theoretical and Applied Climatology, 128, 207-222. https://doi.org/10.1007/s00704-015-1704-7

[24] Wang, G., Wang, D., Trenberth, K., et al. (2017) The Peak Structure and Future Changes of the Relationships between Extreme Precipitation and Temperature. $\mathrm{Na}$ ture Climate Change, 7, 268-274. https://doi.org/10.1038/nclimate3239 